\title{
In situ grazing incidence total scattering: new in situ capabilities for pair distribution function analysis of thin films
}

\author{
A. Dippel ${ }^{1}$, O. Gutowski ${ }^{2}$, M. Roelsgaard ${ }^{3}$, B. Iversen ${ }^{4}$, M. Sturm ${ }^{5}$, M. v. Zimmermann ${ }^{6}$ \\ ${ }^{1}$ Deutsches Elektronen-Synchrotron DESY ${ }^{2}$ Deutsches Elektronen-Synchrotron, ${ }^{3}$ Department of \\ Chemistry, Aarhus University, ${ }^{4}$ Department of Chemistry, Aarhus University, ${ }^{5}$ Deutsches \\ Elektronen-Synchrotron DESY, ${ }^{6}$ Deutsches Elektronen-Synchrotron DESY \\ ann-christin.dippel@desy.de
}

Structural disorder in materials that exhibit local deviations from their periodic structure or no long-range ordering at all is effectively described in terms of the atomic pair distribution function (PDF). A PDF represents the probability of finding pairs of atoms at distances $r$ in real space from nearest-neighbors up to correlations on the nanometer scale. The total scattering (TS) approach that enables PDF analysis requires that scattering data is collected over a wide range in reciprocal space and subsequently a Fourier transformation of the entire scattering pattern into direct space. For bulk-type samples, TS has become a standard routine at high-energy beamlines. On the other hand, its application to thin film with thicknesses ranging from below ten to a few hundred nanometers is challenging since the unfavorable thickness ratio of the layer to its substrate (typically tens to hundreds of micrometers or more) limits the detectability of the film signal in transmission geometry, the usual approach for bulk systems and demonstrated for films as described e.g. in Ref. [1]. Therefore, we applied the high-energy surface diffraction technique established for single-crystal surfaces [2] to less ordered films and successfully expanded PDF analysis to thin films of only a few nanometers at acquisition times of seconds. [3,4] Besides polycrystalline and textured metal and oxide layers, we studied amorphous and naocrystalline thin films. All films were deposited on amorphous substrates (glass) which provide for easy scaling of the background signal and straightforward subtraction from the sample data to isolate the film signal. Real thin film devices e.g. for electronic applications, however, typically consist of multiple layers, and the film properties largely depend on the nature of the underlying layer. By scanning the incidence angle on the order of millidegrees, we obtained depth-resolved PDFs from bilayer samples. In this way, we were able to study the structure of the individual layers of different combinations and stackings of amorphous and crystalline materials exhibiting high and low (electron) density and, hence, x-ray scattering power from $\mathrm{TiO} 2$ to $\mathrm{Pt}$ [5]. As thermal treatment is an essential part of thin film device manufacturing, we are developing a sample stabilization system that keeps the surface in grazing incidence alignment in the micrometer-sized beam and below the critical angle of total external reflection of $>0.05^{\circ}$. Based on a specially designed laser interferometer and an active feedback loop, this system enables following structural changes during variable-temperature processes up to several hundred degrees. This presentation will highlight the new in situ capability by the proof-of-concept experiment of crystallizing a $30 \mathrm{~nm}$ amorphous $\mathrm{HfO} 2$ thin film while continuously acquiring TS data at a time resolution of $0.5 \mathrm{~s}$.

[1] Jensen, K. M. Ø. Blichfeld, A. B. \& Goldman, A. I. (1999). Rev. Sci. Instrum. 70, 3554.

[2] Gustafson, J. Shipilin, M. Zhang, C. Stierle, A. Hejral, U. Ruett, U. Gutowski, O. Carlsson, P.-A. Skoglundh, M. Lundgren, E. (2014). Science 343, 758.

[3] Dippel, A.-C. Roelsgaard, M. Böttger, U. Schneller, T. Gutowski, O. Rütt, U. (2019). IUCrJ 6, 290.

[4] Roelsgaard, M. Dippel, A.-C. Borup, K. A. Nielsen, I. G. Broge, N. L. N. Röh, J. T. Gutowski, O. Iversen, B. B. (2019). IUCrJ 6, 299.

[5] Dippel, A.-C. Gutowski, O. Klemeyer, L. Boettger, U. Berg, F. Schneller, T. Hardtdegen, A. Aussen, S. Hoffmann-Eifert, S. Zimmermann, M. v. (2020). Nanoscale 12, 13103. 